\title{
Statistical Properties of Inelastic Lorentz Gas
}

\author{
$\operatorname{AUTHOR}(S)$ :
}

Kim, Hyeon-Deuk; Hayakawa, Hisao

\section{CITATION:}

Kim, Hyeon-Deuk ... [et al]. Statistical Properties of Inelastic Lorentz Gas. Journal of the Physical Society of Japan 2001, 70(7): 1954-1965

\section{ISSUE DATE:}

2001-07

URL:

http://hdl.handle.net/2433/49786

\section{RIGHT:}

Copyright (c) 2001 The Physical Society of Japan; This is not the published version. Please cite only the published version.; この論文は 出版社版でありません。引用の際には出版社版をご確認ご利用くださ い。 


\title{
Statistical Properties of Inelastic Lorentz Gas
}

\author{
KIM Hyeon-Deuk $^{*}$ and Hisao HAYAKawa ${ }^{\dagger}$ \\ Graduate School of Human and Environmental Studies, Kyoto University, Kyoto 606-8501
}

\begin{abstract}
The inelastic Lorentz gas in cooling states is studied. It is found that the inelastic Lorentz gas is localized and that the mean square displacement of the inelastic Lorentz gas obeys a power of a logarithmic function of time. It is also found that the scaled position distribution of the inelastic Lorentz gas has an exponential tail, while the distribution is close to the Gaussian near the peak. Using a random walk model, we derive an analytical expression of the mean square displacement as a function of time and the restitution coefficient, which well agrees with the data of our simulation. The exponential tail of the scaled position distribution function is also obtained by the method of steepest descent.
\end{abstract}

\footnotetext{
*E-mail:kim@yuragi.jinkan.kyoto-u.ac.jp

${ }^{\dagger}$ E-mail: hisao@yuragi.jinkan.kyoto-u.ac.jp
} 


\section{INTRODUCTION}

The statistical properties of dissipative systems have created considerable attention from the view point of non-equilibrium statistical mechanics. The analysis of gas kinetics has played an important role in the construction of statistical mechanics. Similarly, granular gases which are collections of inelastic particles [1.2] are expected to be important to understand non-equilibrium statistical mechanics of dissipative systems. To understand the statistical properties of dissipative systems, several attempts have been carried out on a collection of inelastic particles.

The simplest model of granular gases is an inelastic hard core model. To analyze the time evolution of the velocity distribution function (VDF), we usually adopt the inelastic Boltzmann equation which is a generalization of the Boltzmann equation to the case of inelastic gases. There have been several investigations on VDF of the inelastic gas by the inelastic Boltzmann equation. [4 6, 3, 7 10] The approximate analytical solution of the inelastic Boltzmann equation has been obtained by the method of expansion by the Sonine polynomial which is known as a fast convergent one and becomes standard technique to describe both non-uniform elastic gases and uniform inelastic gas systems [4 6, 3, 2, 10]. They also suggested that the scaled VDF of the inelastic Boltzmann equation has an exponential high energy tail $[4,6$, [3], which has been confirmed by the direct simulation Monte Carlo method (DSMC) [3] or in molecular dynamics simulations of smooth hard inelastic disks in the homogeneous cooling state (HCS) [⿴囗十 . On the other hand, some investigations on the spatial structure of the inelastic Boltzmann gas system have been performed [6, 11, 12]. Esipov and Pöschel [6] have suggested that the diffusion length of particles obeys a power of a logarithmic function of time. Brey et al. [11] have derived the self-diffusion coefficient by means of a Chapmann-Enskog expansion in the density gradient of the particles in the inelastic Boltzmann gas system. Chen et al. [12] have shown in the molecular dynamics simulation of sticky inelastic particles that the probability density function of inelastic particles obeys an exponential distribution at late stages. 
There is, however, a strong restriction in the applicability of the inelastic Boltzmann equation and the method of the Sonine expansion. Inelastic collisions generate string-like clusters in a cooling state which sometimes reaches an inelastic collapse state. Thus, HCS cannot be kept in general [13 15, 12]. Thus, both of the inelastic Boltzmann equation and the method of the Sonine expansion which are only valid in dilute HCS [4, 5, 3, 7, 10] become no longer valid as time goes on [16].

On the other hand, the inelastic scatterings of classical electrons by ions can be modeled by the inelastic Lorentz gas [17,18] in which few fraction of particles can move freely among many immobile scatters known as Drude's formula. [19] Since the fixed scatters keep their initial configuration, the system can keep the state of dilute mobile particles if the initial configuration is dilute. Furthermore, there are some examples of the inelastic Lorentz gas in the natural phenomena such as the spontaneous interparticle percolation. 17, 18 The Lorentz gas is also familiar in dairy life such as the pedestrian motion among a cloud of persons and obstacles, the pachinko game and the pinball game 20 etc. There is an additional advantage that the analysis of the inelastic Lorentz gas model is easier than that of the inelastic Boltzmann equation because the inelastic Lorentz gas model treats the one-body problem: The situation of Lorentz gas is dilute mobile particles among almost immobile scatters, while Boltzmann gas is a collection of mobile particles.

We believe that the systematic studies of the inelastic Lorentz gas system is also needed. The steady VDF of inelastic Lorentz gas and its percolation velocity under the uniform external field have been analyzed. [17,18 However, there have been no efforts to investigate the spatial structure of the inelastic Lorentz gas system without external field. It is important to discuss the spatial structure of the inelastic Lorentz gas without any external field as the first step of the study of the inelastic Lorentz gas system. Thus, we intend to investigate the spatial structure of the inelastic Lorentz gas in cooling state in this paper.

The organization of this paper is as follows. In $\S 2$, we introduce the model of our simulation. In $\S 3$, we validate our simulation code by evaluating the mean square displacement, the velocity distribution and the position distribution in elastic collisions. We also explain 
the main results of our simulation and analysis for the mean square displacement and the position distribution of the inelastic Lorentz gas in $\S 3$. We conclude our results in $\S 4$.

\section{THE MODEL OF OUR SIMULATION}

In our simulation we adopt the event driven method for hard core collision of particles. 2123 The system consists of 40000 fixed smooth hard disks, that is 40000 scatters, and one tracer disk. The collision between the tracer and the scatters is inelastic hard core collision with a constant coefficient of restitution $e$. Through our simulation, we use dimensionless quantities in which the scales of length and velocity are respectively measured by the diameter of the disks $d$ and the standard deviation of the initial velocity distribution $U$. Namely, both of all the scatters and the tracer are circular disks with their diameter $d$. The number density of the scatters in this system is $0.04 d^{-2}$, where the system size is $L_{x}=L_{y}=1000 d$. Because the area fraction is approximately 0.0314 , the system of our simulation is dilute enough. We adopt the periodic boundary condition in this system. The system size is enough large to remove the boundary effect of this system, because the tracer is localized and have rarely gone across the boundaries. We average each result over 10000 samples which consist of 100 different initial configurations of the scatters and 100 different initial velocities of the tracer. Each initial configuration of the scatters is at random. Each tracer starts from the center of the system and its distribution of the initial velocities is made from normal random numbers, where its average is zero and its standard deviation is $U$. Each sample has been calculated until 100 inelastic collisions.

In each inelastic collision, the velocity of a tracer tangential to the collision plane are preserved, while the velocity normal to it changes with the restitution coefficient $e$ which is assumed to be a constant for all the collisions. The precollision velocity of the tracer $\mathbf{v}^{*}$ yields the postcollision velocity of it $\mathbf{v}$ with $\mathbf{v} \cdot \hat{\sigma}=-e\left(\mathbf{v}^{*} \cdot \hat{\sigma}\right)$, where $\hat{\sigma}$ is a unit vector pointing from the center of the scatter to the center of the tracer ( Fig.1). Thus, the expression is given by 


$$
\mathbf{v}=\mathbf{v}^{*}-(1+e)\left(\mathbf{v}^{*} \cdot \hat{\sigma}\right) \hat{\sigma}
$$

This relation between the precollision velocity and the postcollision velocity in the inelastic Lorentz gas system is quite different from that in the inelastic Boltzmann gas system.

In the simulation of the inelastic Lorentz gas, the velocity of the tracer quickly decreases. For the sake of long time simulation, we adopt the rescaling of the speed of the tracer as follows. After each collision, the magnitude of the postcollision velocity $|\mathbf{v}|$ has been put to that of the precollision velocity $\left|\mathbf{v}^{*}\right|$, i.e. the magnitude of the initial velocity of the tracer has been preserved for all the collisions in each sample. Note that we use non-scaled values of $\mathbf{v}$ the magnitude of which decreases by collisions for our analysis.

\section{RESULTS: SIMULATION AND THEORY}

Now, we explain the main results of our simulation and analysis for inelastic Lorentz gas. We study the mean square displacement and the position distribution of the tracer.

\section{A. Check of our simulation code}

Our simulation code has been validated by the test simulation in which we have evaluated the mean square displacement, the velocity distribution and the position distribution of the tracer in the elastic collisions, i.e. $e=1$. For elastic scatters, we expect that the tracer displays random walk motion when the initial speeds of the tracer are identical and that the Gaussian is the stable velocity distribution.

Corresponding to a random walk model, we have fixed the magnitudes of 100 different initial velocities of the tracer, i.e. $\left|\mathbf{v}_{0}\right|=U$. In each sample, we calculate the motion of tracer until 10000 elastic collisions. The other conditions of the test simulation are same as those of the simulation in inelastic collisions. We have checked that the mean square displacement of the tracer $\left\langle\mathbf{r}^{2}(t)\right\rangle$ is almost a linear function of time, where $\langle\cdots\rangle$ indicates the average 
over the 10000 samples. We also confirm that all the data of the position distribution $g(r, t)$ can be scaled as

$$
g(r, t)=\frac{1}{\sqrt{\left\langle\mathbf{r}^{2}(t)\right\rangle}} G(R),
$$

where $G(R)$ is the scaled position distribution function and $R \equiv r / \sqrt{\left\langle\mathbf{r}^{2}(t)\right\rangle}$ is the scaled distance from the center of the system at which the tracer started. We check that the scaled position distribution $G(R)$ is almost on the Gaussian curve as expected from the conventional random walk. Furthermore, the velocity distribution of the tracer becomes almost the Gaussian.

\section{B. Mean square displacement}

Let us discuss the statistical properties of inelastic Lorentz gas. At first, we evaluate the time evolution of the thermal velocity and discuss the mean square displacement in the inelastic system.

In the inelastic Lorentz gas system, many inelastic collisions happen at random. The average number of collision times per unit time is evaluated as $2 d v_{0}(t) \rho_{0}$, where $d$ is the diameter of the tracer and the scatters and $\rho_{0}$ is the number density of the scatters. The thermal velocity $v_{0}(t)$ is defined as

$$
v_{0}(t) \equiv \sqrt{\frac{1}{n} \int \mathbf{v}^{2} f(\mathbf{v}, t) \mathrm{d} \mathbf{v}}
$$

with the velocity distribution of the tracer $f(\mathbf{v}, t)$ and the average density of the tracer $n \equiv \int f(\mathbf{v}, t) d \mathbf{v}$. Therefore the temperature of the system is denoted as

$$
T(t)=\frac{1}{2} m v_{0}^{2}(t)
$$

where $m$ is the mass of the tracer. [4, 5, 9, 3]

In each collision, the energy loss of the tracer is considered to be proportional to the temperature of the system $T(t)$. Thus, we can estimate the energy loss of the tracer per unit time as 


$$
\frac{\mathrm{d} T(t)}{\mathrm{d} t}=-2 d v_{0}(t) \rho_{0} h(e) T(t)
$$

where $h(e)$ is a dimensionless function of the restitution coefficient $e$. $h(e)$ represents a cooling rate of the inelastic Lorentz gas system. Substitution of eq. (4) into eq. (5) leads to

$$
\frac{\mathrm{d} v_{0}(t)}{\mathrm{d} t}=-d \rho_{0} h(e) v_{0}^{2}(t)
$$

Solving eq. (6), we obtain the thermal velocity $v_{0}(t)$ as

$$
v_{0}(t)=\frac{1}{\frac{1}{v_{0}(0)}+d \rho_{0} h(e) t},
$$

where $v_{0}(0)$ is the initial thermal velocity. In the inelastic Boltzmann gas system, Haff [24] has derived the same expression as that of eq.(7), where the cooling rate is assumed to be proportional to $1-e^{2}$.

Here, we can deduce the expression of $h(e)$ from our simulation as follows. Figure 2 shows $\left(v_{0}^{-1}(t)-v_{0}^{-1}(0)\right) /(1-e) U$ versus $t U d^{-1}$ plots for several values of the restitution coefficient, namely, for $e=0.4,0.6,0.7$ and 0.9. All the data are on the same straight line which is also shown in Fig.2. Corresponding to the results of our simulation, we set the number density of the scatters $\rho_{0}=0.04 d^{-2}$ and the initial thermal velocity $v_{0}(0)=\sqrt{2} U$ from eq.(3). Thus, Fig.2 indicates that $h(e)$ is a linear function of the restitution coefficient $e$ as

$$
h(e) \simeq 1.45 \times(1-e)
$$

whereas Martin and Piasecki [18 have assumed energy losses at collisions in inelastic Lorentz gas system are proportional to $1-e^{2}$. This Haff's law, $h(e) \sim 1-e^{2}$, can be derived from the Gaussian VDF. We will demonstrate that the cooling rate of the inelastic Lorentz gas system is not proportional to $1-e^{2}$ and close to eq.(8) in terms of non-Gaussian nature of VDF as follows.

The time evolution of VDF for inelastic Lorentz gas without any external field is in general described by a rate equation. For elastic gases, the rate equation is known as the 
Boltzmann equation. Similarly we can derive a rate equation of VDF for inelastic Lorentz gas systems (see Appendix A), i.e. the inelastic Lorentz gas equation :

$$
\frac{\partial f(\mathbf{v}, t)}{\partial t}=d \rho_{0} \int_{\mathbf{v} \cdot \hat{\sigma}>0}(\mathbf{v} \cdot \hat{\sigma})\left[\frac{f\left(\mathbf{v}^{*}, t\right)}{e^{2}}-f(\mathbf{v}, t)\right] \mathrm{d} \hat{\sigma} .
$$

We assume that eq.(9) admits an scaling solution depending on time only through the thermal velocity $v_{0}(t)$ defined in eq.(邽) as

$$
f(\mathbf{v}, t)=\frac{n}{v_{0}^{2}(t)} F(\mathbf{c})
$$

with the scaled velocity of a tracer $\mathbf{c} \equiv \mathbf{v} / v_{0}(t)$ and the average density of the tracer $n \equiv \int f(\mathbf{v}, t) d \mathbf{v} . F(\mathbf{c})$ is the scaled VDF of the tracer which is independent of time. Here we define the dimensionless collision integral as

$$
I(F) \equiv \int_{\mathbf{c} \cdot \hat{\sigma}>0}(\mathbf{c} \cdot \hat{\sigma})\left[\frac{F\left(\mathbf{c}^{*}\right)}{e^{2}}-F(\mathbf{c})\right] \mathrm{d} \hat{\sigma},
$$

and its moments as

$$
M_{p} \equiv-\int c^{p} I(F) \mathrm{d} \mathbf{c}
$$

Multiplying eq.(9) by $m \mathbf{v}^{2} / 2$ and integrating by $\mathbf{v}$, we obtain the rate of change of the temperature from eqs. (3), (4), (11), and (12) as

$$
\frac{\mathrm{d} T(t)}{\mathrm{d} t}=-d v_{0}(t) \rho_{0} M_{2} T(t) .
$$

Comparing eq.(13) with eq. (5), we obtain

$$
h(e)=\frac{M_{2}}{2} .
$$

Both sides of eq.(14) represent the cooling rate of the inelastic Lorentz gas system. When we adopt the lowest order Sonine expansion in eq.(B4), the expression of $M_{2}$ as a function of the restitution coefficient $e$ is given as

$$
M_{2}=\sqrt{\pi}\left(1-e^{2}\right)\left(\frac{65}{64}+\frac{15}{32} e^{2}-\frac{1}{64} \sqrt{-383+828 e^{2}+900 e^{4}}\right) .
$$


The details of the derivation of eq.(B27) are written in Appendix B. Figure 3 shows the both sides of eq.(14), i.e. $M_{2} / 2$ from eq.(B27) and $h(e)$ from eq.(8) which has been estimated from the data of our simulation, as a function of the restitution coefficient $e$. There are no significant differences between $M_{2} / 2$ and $h(e)$, though $M_{2} / 2$ does not exist in the high inelastic region. This means that Haff's law, $h(e) \sim 1-e^{2}$, may be replaced by eq.(8) in the inelastic Lorentz gas system as a result of non-Gaussian nature.

Now, let us discuss the statistical properties of the inelastic Lorentz gas based on a random walk model. To simplify the expressions, we introduce $\alpha$ and $\beta$ as,

$$
\alpha \equiv \frac{1}{v_{0}(0)}, \quad \beta \equiv d \rho_{0} h(e)
$$

Using $\alpha$ and $\beta$, the average time interval $\tau_{N}$ from $N-1$ th collision to $N$ th collision between the tracer and the scatters is given as,

$$
\tau_{N} \simeq \frac{l}{v_{0}\left(t_{N-1}\right)}=l\left(\alpha+\beta t_{N-1}\right)
$$

where $l$ denotes the mean free path of the tracer, and $t_{N-1}$ is the total time until the $N-1$ th collision. From the relation $t_{N-1}=\tau_{1}+\tau_{2}+\cdots+\tau_{N-1}$, we obtain $\tau_{N}$ as a function of the number of collision times $N$,

$$
\tau_{N}=l \alpha(1+l \beta)^{N-1}
$$

Therefore the total time until the $N$ th collision $t_{N}$ is given by

$$
t_{N}=\tau_{1}+\tau_{2}+\cdots+\tau_{N}=\frac{\alpha}{\beta}\left\{(1+l \beta)^{N}-1\right\} .
$$

Rearranging eq.(19), the number of collision $N$ is obtained as a function of the total time until the $N$ th collision $t_{N}$ as

$$
N=\frac{\ln \left(\frac{\beta}{\alpha} t_{N}+1\right)}{\ln (1+l \beta)} .
$$

Note that eq.(20) holds even for the elastic limit

$$
N \rightarrow \frac{t_{N}}{\alpha l} \quad \text { for } \quad \mathrm{e} \rightarrow 1
$$


Here we have used the result of eq.(8), i.e. $h(e) \rightarrow 0$ for $e \rightarrow 1$.

If we assume that the inelastic Lorentz gas system corresponds to the random walk system with the reduction of hopping velocity, the effect of inelasticities can be absorbed in the number of collisions $N$. Thus, we deduce the expression of mean square displacement of a tracer as

$$
\left\langle\mathbf{r}^{2}(t)\right\rangle_{e}=l^{2} N \simeq l^{2} \frac{\ln \left(\frac{\beta}{\alpha} t+1\right)}{\ln (1+l \beta)}
$$

where we use eq.(20) and the suffix $e$ of the bracket emphasizes the quantity with the restitution coefficient $e$. Let us evaluate some physical quantities in eq.(22). Using the values of our simulation, we obtain $\alpha=1 / \sqrt{2} U^{-1}, \beta \simeq 0.058 \times(1-e) d^{-1}$ from eqs.(8) and (16). We set the mean free path of the tracer as $l \simeq 13.5 d$ instead of the theoretical mean free path $l=12.5 d$.

Substituting these physical quantities and eq.(8) into eq.(22), we obtain the mean square displacement as a function of time and restitution coefficient. Figure $\operatorname{tshows}\left\langle\mathbf{r}^{2}(t)\right\rangle_{e} \times \ln (1+$ $l \beta) d^{-2}$ versus $(1-e) t U d^{-1}$ plots for $e=0.4,0.6,0.7$ and 0.9 . All the results of our simulation shown as the symbols are on the same curve. Our results clearly demonstrate that the tracer is localized in the case $e<1$ and that the mean square displacement seems to obey a power of the logarithmic function of time. It should be emphasized that eq.(22), shown in Fig.4, becomes identical with the results of our simulation as time goes on. The reason why eq.(22) deviates a little from the data of our simulation in the early time region in Fig. 1 is that few collisions have happened in this region.

\section{Position distribution}

In the inelastic Lorentz gas system, the position distribution function of the tracer $g_{e}(r, t)$ for the restitution coefficient $e$ is scaled by the mean square displacement $\left\langle\mathbf{r}^{2}(t)\right\rangle_{e}$. The scaled position distribution function of the tracer $G(R)$ is defined as

$$
G(R) \equiv \sqrt{\left\langle\mathbf{r}^{2}(t)\right\rangle_{e}} \times g_{e}(r, t)
$$


where $R \equiv r / \sqrt{\left\langle\mathbf{r}^{2}(t)\right\rangle_{e}}$ is the scaled distance from the center of the system at which the tracer started. $G(R)$ is independent of the restitution coefficient $e$ and time. Let us study the scaled position distribution of the tracer $G(R)$. Figure 5 shows the results of our simulation and analysis. The symbols are results of our simulation which show the scaled position distribution of the tracer for $e=0.4,0.6,0.7$ and 0.9 at four different times $t=3000 d U^{-1}, 300 d U^{-1}, 500 d U^{-1}$ and $1000 d U^{-1}$, respectively. All the data of the scaled position distribution $G(R)$ are almost on the universal curve. It should be noted that the curve is close to a Gaussian near its peak, while the tail seems to obey an exponential function. This exponential tail has been observed in a simulation of granular gas system. 12

On the other hand, the broken line and the dash-dotted line in Fig.5 are results of our theoretical analysis which will be explained later. The broken line corresponds to the data in the tail region of the scaled position distribution, while the dash-dotted line corresponds to the data in the peak region of the scaled position distribution. Theoretical curves well explain the result of our simulation. Now, let us explain how to obtain the theoretical curves.

At first, we assume that the tracer obeys a renormalized diffusive motion as assumed in the previous subsection. This assumption is validated by the derivation of the diffusion equation of the tracer by means of a Chapman-Enskog expansion in the density gradient of the tracer. [10,11] The time evolution of the position-velocity distribution of the tracer $p(\mathbf{r}, \mathbf{v}, t)$ is given by the inelastic Lorentz gas equation (see Appendix A):

$$
\frac{\partial p(\mathbf{r}, \mathbf{v}, t)}{\partial t}+\mathbf{v} \cdot \nabla p(\mathbf{r}, \mathbf{v}, t)=\Lambda p(\mathbf{r}, \mathbf{v}, t)
$$

where the operator $\Lambda$ is defined as

$$
\Lambda p(\mathbf{r}, \mathbf{v}, t)=d \rho_{0} \int_{\mathbf{v} \cdot \hat{\sigma}>0}(\mathbf{v} \cdot \hat{\sigma})\left[\frac{p\left(\mathbf{r}, \mathbf{v}^{*}, t\right)}{e^{2}}-p(\mathbf{r}, \mathbf{v}, t)\right] \mathrm{d} \hat{\sigma}
$$

Integrating this equation by $\mathbf{v}$, we obtain

$$
\frac{\partial n(\mathbf{r}, t)}{\partial t}+\nabla \cdot \mathbf{J}(\mathbf{r}, t)=0
$$

where the density of the tracer $n(\mathbf{r}, t)$ is defined as 


$$
n(\mathbf{r}, t)=\int \mathrm{d} \mathbf{v} p(\mathbf{r}, \mathbf{v}, t)
$$

and the flux of the tracer $\mathbf{J}(\mathbf{r}, t)$ is defined as

$$
\mathbf{J}(\mathbf{r}, t)=\int \mathrm{d} \mathbf{v} \mathbf{v} p(\mathbf{r}, \mathbf{v}, t)
$$

Note that the integral of the right hand side of eq.(24) by the velocity of the tracer becomes zero, because it denotes the effect of change in the velocity of the tracer.

Here we adopt the Chapman-Enskog method in which the position-velocity distribution of the tracer $p(\mathbf{r}, \mathbf{v}, t)$ is written as a series expansion in a formal parameter $\epsilon$,

$$
p(\mathbf{r}, \mathbf{v}, t)=p^{(0)}(\mathbf{r}, \mathbf{v}, t)+\epsilon p^{(1)}(\mathbf{r}, \mathbf{v}, t)+\epsilon^{2} p^{(2)}(\mathbf{r}, \mathbf{v}, t)+\cdots .
$$

$\epsilon$ implies an implicit $\nabla n(\mathbf{r}, t)$ which provokes diffusion of the tracer. Consistently, eq. 26) is expanded as

$$
\frac{\partial n(\mathbf{r}, t)}{\partial t}=\sum_{j=0}^{\infty} \epsilon^{j+1} \partial_{t}^{(j)} n(\mathbf{r}, t)
$$

with

$$
\partial_{t}^{(j)} n(\mathbf{r}, t)=-\nabla^{(0)} \cdot \mathbf{J}^{(j)}(\mathbf{r}, t)
$$

and

$$
\mathbf{J}^{(j)}(\mathbf{r}, t)=\int \mathrm{d} \mathbf{v} \mathbf{v} p^{(j)}(\mathbf{r}, \mathbf{v}, t)
$$

Note that

$$
\nabla=\epsilon \nabla^{(0)}+\epsilon^{2} \nabla^{(1)}+\cdots
$$

The zeroth order of eq.(24), thus, becomes

$$
\frac{\partial T(t)}{\partial t} \frac{\partial p^{(0)}(\mathbf{r}, \mathbf{v}, t)}{\partial T(t)}=\Lambda p^{(0)}(\mathbf{r}, \mathbf{v}, t)
$$

A comparison of this with eq.(9) indicates that $p^{(0)}(\mathbf{r}, \mathbf{v}, t)$ is proportional to $f(\mathbf{v}, t)$. Owing to the normalization, 


$$
p^{(0)}(\mathbf{r}, \mathbf{v}, t)=\frac{n(\mathbf{r}, t)}{n} f(\mathbf{v}, t) .
$$

Using this relation and eq.(32), $\mathbf{J}^{(0)}(\mathbf{r}, t)=0$, i.e. the flux of the tracer vanishes to zeroth order, which leads to $\partial_{t}^{(0)} n(\mathbf{r}, t)=0$. Additionally, from eq.(35), $\partial p^{(0)}(\mathbf{r}, \mathbf{v}, t) / \partial n(\mathbf{r}, t)=$ $f(\mathbf{v}, t) / n$. To the first order in the density gradient $\epsilon$, eq.(24) leads to

$$
\begin{aligned}
& \left(\partial_{t}^{(0)} n(\mathbf{r}, t)\right) \frac{\partial p^{(0)}(\mathbf{r}, \mathbf{v}, t)}{\partial n(\mathbf{r}, t)}+\frac{\partial T(t)}{\partial t} \frac{\partial p^{(1)}(\mathbf{r}, \mathbf{v}, t)}{\partial T(t)} \\
& +\left(\mathbf{v} \cdot \nabla^{(0)} n(\mathbf{r}, t)\right) \frac{\partial p^{(0)}(\mathbf{r}, \mathbf{v}, t)}{\partial n(\mathbf{r}, t)}=\Lambda p^{(1)}(\mathbf{r}, \mathbf{v}, t) .
\end{aligned}
$$

Using the results of the zeroth order and eq.(5), this equation becomes

$$
\left(\xi(t) T(t) \frac{\partial}{\partial T(t)}+\Lambda\right) p^{(1)}(\mathbf{r}, \mathbf{v}, t)=\frac{\left(\mathbf{v} \cdot \nabla^{(0)} n(\mathbf{r}, t)\right)}{n} f(\mathbf{v}, t)
$$

with $\xi(t)=2 d v_{0}(t) \rho_{0} h(e)$. The solution $p^{(1)}(\mathbf{r}, \mathbf{v}, t)$ is proportional to $\nabla^{(0)} n(\mathbf{r}, t)$ :

$$
p^{(1)}(\mathbf{r}, \mathbf{v}, t)=\mathbf{B}(\mathbf{v}, T(t)) \cdot \nabla^{(0)} n(\mathbf{r}, t),
$$

where the vector $\mathbf{B}(\mathbf{v}, T(t))$ also depends on $t$ through $T(t)$. Substitution of this into eq.(37) leads to

$$
\left(\xi(t) T(t) \frac{\partial}{\partial T(t)}+\Lambda\right) \mathbf{B}(\mathbf{v}, T(t))=\frac{\mathbf{v}}{n} f(\mathbf{v}, t) .
$$

Here, the contribution to the particle flux of first order in the density gradient is given by

$$
\mathbf{J}^{(1)}(\mathbf{r}, t)=-D_{e}(t) \nabla^{(0)} n(\mathbf{r}, t),
$$

with

$$
D_{e}(t) \equiv-\frac{1}{2} \int \mathrm{d} \mathbf{v} \mathbf{v} \cdot \mathbf{B}(\mathbf{v}, T(t))
$$

Here we have used symmetry considerations in two-dimension. Using the diffusion coefficient $D_{e}(t)$, eq.(41) becomes

$$
\left(\xi(t) T(t) \frac{\partial}{\partial T(t)}-\nu_{D}\right) D_{e}(t)=-\frac{T}{m},
$$


with

$$
\nu_{D} \equiv-\frac{\int(\Lambda \mathbf{B}) \cdot \mathbf{v d} \mathbf{v}}{\int \mathbf{B} \cdot \mathbf{v} \mathrm{d} \mathbf{v}}
$$

Because $\xi(t) \sim T^{\frac{1}{2}}(t)$, dimensional analysis requires $D_{e}(t) \sim T^{\frac{1}{2}}(t)$. Therefore,

$$
\frac{\partial D_{e}(t)}{\partial T(t)}=\frac{D_{e}(t)}{2 T(t)}
$$

Substitution of this into eq.(42) becomes

$$
D_{e}(t)=\frac{T(t)}{m}\left(\nu_{D}-\frac{\xi(t)}{2}\right)^{-1}
$$

From eqs.(40) and (31), we obtain the diffusion equation of the tracer,

$$
\partial_{t}^{(1)} n(\mathbf{r}, t)=D_{e}(t) \nabla^{(0)^{2}} n(\mathbf{r}, t)
$$

with the diffusion coefficient $D_{e}(t)$ in eq.(45). The expression of the diffusion coefficient $D_{e}(t)$ is obtained explicitly in eq. (C7) with the aid of eq.(B18) by the method of the Sonine expansion, though the diffusion coefficient $D_{e}(t)$ in eq.(C7) gives a little deviated results from the results of our simulation in the inelastic Lorentz gas system as shown in Appendix C. Now the random walk picture is validated by this standard method.

Therefore, the tracer obeys a renormalized diffusive motion; the original position distribution function of the tracer $\tilde{g}_{e}(r, t)$ is expected to be given by

$$
\tilde{g}_{e}(r, t) \simeq \frac{1}{4 \int_{0}^{t} D_{e}\left(V, t^{\prime}\right) \mathrm{d} t^{\prime}} r \exp \left[-\frac{r^{2}}{8 \int_{0}^{t} D_{e}\left(V, t^{\prime}\right) \mathrm{d} t^{\prime}}\right] .
$$

Here we have taken into account the distribution of hopping velocity; we have adopted the diffusion coefficient as

$$
D_{e}\left(V, t^{\prime}\right) \equiv \frac{\kappa V^{2} l}{\alpha+\beta t^{\prime}}
$$

where $\kappa$ is undetermined dimensionless coefficient which will not be indicated explicitly, $\tau$ is time interval between two adjacent collisions, $\mathbf{l}$ is the hopping vector of the tracer, and $V$ is the magnitude of the scaled hopping velocity defined as 


$$
V \equiv\left|\frac{1 / \tau}{v_{0}\left(t^{\prime}\right)}\right|
$$

The expression of eq.(48) is derived in Appendix D. Introducing $\eta$ as

$$
\eta \equiv \frac{4 \int_{0}^{t} D_{e}\left(V, t^{\prime}\right) \mathrm{d} t^{\prime}}{V^{2}\left\langle\mathbf{r}^{2}(t)\right\rangle_{e}}
$$

we obtain the expression of $\eta$ from eq. (22) as

$$
\eta=\frac{4 \kappa \ln (1+l \beta)}{l \beta}
$$

which is independent of time. Thus, from eqs.(47) and (50), we can derive the original scaled position distribution function of the tracer $\tilde{G}(R, V)$ as

$$
\tilde{G}(R, V)=\frac{1}{\eta V^{2}} R \mathrm{e}^{-\frac{R^{2}}{2 \eta V^{2}}}
$$

Here we assume the scaled hopping velocity corresponds to the scaled velocity of the tracer $\mathbf{c} \equiv \mathbf{v} / v_{0}(t)$. In our simulation, all the data of the scaled velocity distribution of the tracer $F(\mathbf{c})$ can be fitted by the Maxwell-Boltzmann distribution $\pi^{-1} \mathrm{e}^{-\mathbf{c}^{2}}$ as Fig.6 shows, though the high energy tail of the tracer deviates from the Gaussian (see Appendix B). Therefore, we assume the distribution of the scaled hopping velocity is almost Gaussian. Now, the scaled position distribution function of the tracer $G(R)$ is expressed by

$$
G(R)=\int_{0}^{\infty} \frac{\mathrm{e}^{-V^{2}}}{\pi} \frac{1}{\eta V^{2}} R \mathrm{e}^{-\frac{R^{2}}{2 \eta V^{2}}} 2 \pi V \mathrm{~d} V=\frac{2}{\eta} R \int_{0}^{\infty} \mathrm{e}^{-p(V, R) R^{2}} \mathrm{~d} V .
$$

The second equality in eq.(53) defines $p(V, R)$ as

$$
p(V, R) \equiv \frac{\ln V}{R^{2}}+\frac{V^{2}}{R^{2}}+\frac{1}{2 \eta V^{2}}
$$

We analyze eq.(53) by the method of steepest descent in order to obtain the tail region of the scaled position distribution function. Designating $V$ which satisfies $\partial p(V, R) / \partial V=0$ as $V^{*}(R)$, the expression of $V^{*}(R)$ is

$$
V^{*}(R)=\sqrt{-\frac{1}{4}+\frac{\sqrt{\eta+8 R^{2}}}{4 \sqrt{\eta}}}
$$


By the method of steepest descent, from eq.(53) we can derive the approximate $G(R)$, i.e.

$$
\begin{aligned}
G(R) & \simeq \frac{2}{\eta} R \int_{0}^{\infty} \exp \left[-p\left(V^{*}, R\right) R^{2}-\left.\frac{\left(V-V^{*}\right)^{2}}{2} \frac{\partial^{2} p(V, R)}{\partial V^{2}}\right|_{V=V^{*}} R^{2}\right] \mathrm{d} V \\
& =\frac{2}{\eta} \sqrt{\frac{\pi}{\left.2 \frac{\partial^{2} p(V, R)}{\partial V^{2}}\right|_{V=V^{*}}}} \mathrm{e}^{-p\left(V^{*}, R\right) R^{2}} .
\end{aligned}
$$

Equation (56) is reduced to

$$
G(R) \simeq \sqrt{\frac{\pi R}{2 \sqrt{2} \eta^{\frac{3}{2}}}} \mathrm{e}^{-\sqrt{\frac{2}{\eta}} R}
$$

in the limit of $R \rightarrow \infty$. Thus, we have shown that the tail of $G(R)$ obeys an exponential law. Now we use

$$
G(R)=\frac{2 \gamma}{\eta} \sqrt{\frac{\pi}{\left.2 \frac{\partial^{2} p(V, R)}{\partial V^{2}}\right|_{V=V^{*}}}} \mathrm{e}^{-p\left(V^{*}, R\right) R^{2}},
$$

instead of eq.(56). Here $\gamma$ is a fitting parameter. We estimate $\eta \simeq 0.25$ and $\gamma \simeq 7.39$ from the comparison of eq. (58) and the data of our simulation on the scaled position distribution in the tail region.

In order to obtain the peak region of the scaled position distribution function, we replace $V$ in eq. (52) by constant $\bar{V}$. Thus, eq.(53) becomes

$$
G(R) \simeq \frac{1}{\eta \bar{V}^{2}} R \mathrm{e}^{-\frac{R^{2}}{2 \eta \bar{V}^{2}}}
$$

Thus, we expect the Gaussian near the peak. Adopting the most probable $V$ as $\bar{V}$, i.e. $\bar{V}=1 / \sqrt{2}, \eta$ is estimated to be 0.75 .

In Fig.5, eq.(58) with $\eta=0.25$ and $\gamma=7.39$ is expressed by the broken line, while eq.(59) with $\eta=0.75$ and $\bar{V}=1 / \sqrt{2}$ is expressed by the dash-dotted line. Figure 5 shows that eq.(58) is in good agreement with the simulation data of the scaled position distribution in the tail region, while the data of our simulation on the scaled position distribution in the peak region are well-fitted by eq.(59). 


\section{DISCUSSION}

Some approximate analytical solutions of the inelastic Boltzmann equation obtained by the method of the Sonine expansion, namely scaled VDF of a granular gas, are in good agreement with the results of DSMC in which HCS holds [3, ], 8] or of the molecular dynamics simulations of smooth hard inelastic disks in HCS [4]. However, it has been also demonstrated that the Sonine expansion diverges if the inelasticity is high in the inelastic Boltzmann gas system. [4] Solutions by the Sonine expansion cannot express the definite exponential form in the high energy tail, because the expression of eq.(B4) does not have terms of odd power of $\mathbf{c}$, while the definite exponential form contains odd power of $\mathbf{c}$. This means that we cannot express the scaled velocity distribution of the inelastic Boltzmann gas system of high inelasticity by eq. (B⿺辶) even if we obtain the perfect form of eq. (B4). The reason why $a_{2}$ does not exist in the inelastic Lorentz gas system of high inelasticity (cf. eq. (B26) ) is thought to be the same reason mentioned above: the solution by the Sonine expansion does not have terms of odd power of $\mathbf{c}$ and it cannot express the scaled velocity distribution of the inelastic Lorentz gas system of high inelasticity because its high energy tail is definitely exponential. Furthermore, in both of the inelastic Boltzmann gas system and the inelastic Lorentz gas system, some of the scaled velocity distributions obtained by the method of the Sonine expansion become negative for large c, which is obviously an odd result.

We assume the scaled VDF of the tracer obeys the Gaussian distribution in $\S 3.3$, while we have derived the non-Gaussian scaled VDF of the tracer in Appendix B. Though Fig. 6 shows the scaled VDF of the tracer seems to obey the Gaussian, its high energy tail is thought to become exponential. The Gaussian scaled VDF shown in Fig. 6 is rationalized by the insufficiency of the number of the samples in our simulation. The non-Gaussianity of the scaled VDF is not essential for the exponential tail of the scaled position distribution function of the tracer, while it is essential for the fact that the cooling rate is not proportional to $1-e^{2}$ but close to $1-e$. However, the cooling rate is almost proportional to $1-e^{2}$ in the inelastic 
Boltzmann system in which the scaled VDF is non-Gaussian. The difference is considered to be due to the difference between one-body problem and many-body problem, i.e. the collision relation between precollision velocity and postcollision velocity of the inelastic Lorentz gas system is quite different from that of the inelastic Boltzmann gas system and the mean free time of the inelastic Lorentz gas system also shows different dependence on time from that of the inelastic Boltzmann gas system.

We have analyzed the spatial structure of the inelastic Lorentz gas system by both of the method of the Sonine expansion and the random walk model. The mean square displacement of the tracer by the random walk model is identical with the results of our simulation, while the mean square displacement by the method of the Sonine expansion does not agree well with them. Furthermore, the exponential tail of the scaled position distribution function of the tracer cannot be derived by the method of the Sonine expansion. Thus, to analyze the inelastic Lorentz gas system, the random walk model is considered to work better than the method of the Sonine expansion, though the random walk model also has some problems, e.g. we have used the mean free path of the tracer which is a little different from the theoretical mean free path.

\section{CONCLUSIONS}

We have found that the mean square displacement of the inelastic Lorentz gas obeys a power of the logarithmic function of time and that the scaled position distribution of the inelastic Lorentz gas has an exponential tail, while the peak is the Gaussian. We have analytically derived the mean square displacement as a function of time and restitution coefficient which is identical with the data of our simulation. We also analytically obtained the scaled position distribution function the tail of which is exponential and the peak of which is the Gaussian. 


\section{ACKNOWLEDGMENTS}

The author deeply appreciate S. Takesue, Ooshida. T, K. Ichiki and H. Tomita for the useful comments. This study is partially supported by Grant-in-Aid for Science Research Fund from the Ministry of Education, Science and Culture (Grant No. 11740228).

\section{APPENDIX A: TWO-DIMENSIONAL INELASTIC LORENTZ GAS EQUATION}

We explain the derivation of two-dimensional inelastic Lorentz gas equation which can not be derived directly from the inelastic Boltzmann equation.

We designate the position-velocity distribution function of the tracer at time $t$ as $p(\mathbf{r}, \mathbf{v}, t)$. The change of $p(\mathbf{r}, \mathbf{v}, t)$ during minute time $\delta t$ is expressed as

$$
[p(\mathbf{r}, \mathbf{v}, t+\delta t)-p(\mathbf{r}, \mathbf{v}, t)] \mathrm{d} \mathbf{r} \mathrm{d} \mathbf{v}=-\Gamma_{-}+\Gamma_{+}+\Gamma_{\text {flow }}
$$

where $\Gamma_{+}$is the gain term of $p(\mathbf{r}, \mathbf{v}, t)$ by collisions during the minute time, while $\Gamma_{-}$is the loss term of $p(\mathbf{r}, \mathbf{v}, t)$ by collisions during the minute time. $\Gamma_{\text {flow }}$ indicates the change in $p(\mathbf{r}, \mathbf{v}, t)$ due to the flow into or out of $d \mathbf{r}$, free-streaming term, which is given by

$$
\Gamma_{\text {flow }}=-\operatorname{d} \mathbf{r} \mathrm{d} \mathbf{v} \delta t(\mathbf{v} \cdot \nabla) p(\mathbf{r}, \mathbf{v}, t) .
$$

$\Gamma_{-}$and $\Gamma_{+}$are defined as

$$
\Gamma_{-}=\int p(\mathbf{r}, \mathbf{v}, t) \mathrm{d} \mathbf{r} \mathrm{d} \mathbf{v} \rho_{0}|\mathbf{v}| \delta t \mathrm{~d} b
$$

and

$$
\Gamma_{+}=\int p\left(\mathbf{r}, \mathbf{v}^{*}, t\right) \mathrm{d} \mathbf{r} \mathrm{d} \mathbf{v}^{*} \rho_{0}\left|\mathbf{v}^{*}\right| \delta t \mathrm{~d} b=\int p\left(\mathbf{r}, \mathbf{v}^{*}, t\right) \mathrm{d} \mathbf{r} \frac{\mathrm{d} \mathbf{v}}{e} \rho_{0}\left|\mathbf{v}^{*}\right| \delta t \mathrm{~d} b
$$

respectively. Here $\mathbf{v}^{*}$ denotes precollision velocity of the tracer, $\rho_{0}$ is the mean number density of scatters, $e$ is the restitution coefficient, $b$ indicates the impact parameter ( see Fig.7). Note that we use the Jacobian $\mathrm{d} \mathbf{v}^{*}=\mathrm{d} \mathbf{v} / e$. Substitution of eqs.(A3),(A4) and (A2) into eq.(A1) leads to 


$$
\frac{\partial p(\mathbf{r}, \mathbf{v}, t)}{\partial t}+(\mathbf{v} \cdot \nabla) p(\mathbf{r}, \mathbf{v}, t)=\rho_{0} \int\left[\left|\mathbf{v}^{*}\right| \frac{p\left(\mathbf{r}, \mathbf{v}^{*}, t\right)}{e}-|\mathbf{v}| p(\mathbf{r}, \mathbf{v}, t)\right] \mathrm{d} b
$$

It is often convenient to rewrite the integral over the impact parameter as the unit vector $\hat{\sigma}$ as

$$
|\mathbf{v}| \mathrm{d} b=B(|\mathbf{v}|, \hat{\sigma}) \mathrm{d} \hat{\sigma}
$$

Here $\mathrm{d} \hat{\sigma}=\mathrm{d}(\pi-\psi)$ and $b=d \sin (\pi-\psi)$ ( see Fig. 母). $d$ is the diameter of the tracer and the scatters. Thus, the expressions of $B(|\mathbf{v}|, \hat{\sigma})$ and $B\left(\left|\mathbf{v}^{*}\right|, \hat{\sigma}\right)$ are given by

$$
B(|\mathbf{v}|, \hat{\sigma})=|\mathbf{v}| d \cos (\pi-\psi)=d(\mathbf{v} \cdot \hat{\sigma})
$$

and

$$
B\left(\left|\mathbf{v}^{*}\right|, \hat{\sigma}\right)=\left|\mathbf{v}^{*}\right| d \cos (\pi-\psi)=-d\left(\mathbf{v}^{*} \cdot \hat{\sigma}\right)=d \frac{(\mathbf{v} \cdot \hat{\sigma})}{e}
$$

respectively ( see Fig.7 ). From eqs.(A5), (A6), (A7) and (A8), we obtain

$$
\frac{\partial p(\mathbf{r}, \mathbf{v}, t)}{\partial t}+(\mathbf{v} \cdot \nabla) p(\mathbf{r}, \mathbf{v}, t)=d \rho_{0} \int_{\mathbf{v} \cdot \hat{\sigma}>0}(\mathbf{v} \cdot \hat{\sigma})\left[\frac{p\left(\mathbf{r}, \mathbf{v}^{*}, t\right)}{e^{2}}-p(\mathbf{r}, \mathbf{v}, t)\right] \mathrm{d} \hat{\sigma} .
$$

This is nothing but the two-dimensional inelastic Lorentz gas equation. Integrating this equation by $\mathbf{r}$, we obtain eq.(9).

\section{APPENDIX B: COOLING RATE $M_{2}$}

We derive the expression of $M_{2}$ by the method of Sonine expansion.

From eq.(4), eq.(13) is rearranged as

$$
\frac{\mathrm{d} v_{0}(t)}{\mathrm{d} t}=-\frac{\rho_{0} d M_{2} v_{0}^{2}(t)}{2}
$$

Using eqs.(9),(10) and (B1), we obtain the scaled equation for the inelastic Lorentz gas as

$$
I(F)=\frac{M_{2}}{2}\left(2+\mathbf{c} \cdot \frac{\partial}{\partial \mathbf{c}}\right) F(\mathbf{c})
$$

Integration of the product of $c^{p}$ and eq.(B2) by $\mathbf{c}$ makes 


$$
M_{p}=\frac{M_{2}}{2} p\left\langle c^{p}\right\rangle
$$

where $\langle\cdots\rangle$ denotes an average over $F(\mathbf{c})$.

In the limit of small dissipation, $F(\mathbf{c})$ approaches a Gaussian, i.e. $F(\mathbf{c}) \simeq \phi(\mathbf{c}) \equiv$ $\pi^{-1} \exp \left(-\mathbf{c}^{2}\right)$. Therefore, in the low inelasticity region, a systematic approximation of $F(\mathbf{c})$ may be expressed by the Sonine expansion [4, 0, 0, 3, 9, 11, i.e.

$$
F(\mathbf{c})=\phi(\mathbf{c})\left\{1+\sum_{p=1}^{\infty} a_{p} S_{p}\left(\mathbf{c}^{2}\right)\right\}
$$

The first few two-dimensional Sonine polynomials are

$$
\begin{aligned}
& S_{0}(x)=1 \\
& S_{1}(x)=-x+1 \\
& S_{2}(x)=\frac{1}{2} x^{2}-2 x+1 .
\end{aligned}
$$

It should be noted that Sonine polynomials satisfy the orthogonality relations

$$
\int \mathrm{d} \mathbf{c} \phi(\mathbf{c}) S_{p}\left(\mathbf{c}^{2}\right) S_{p^{\prime}}\left(\mathbf{c}^{2}\right)=\delta_{p p^{\prime}}
$$

where $\delta_{p p^{\prime}}$ is the Kronecker delta. From these orthogonality relations, we have

$$
a_{p}=\int \mathrm{d} \mathbf{c} S_{p}\left(\mathbf{c}^{2}\right) F(\mathbf{c})=\left\langle S_{p}\left(\mathbf{c}^{2}\right)\right\rangle .
$$

From eqs.(B6), (B) and (Bי), $a_{1}$ and $a_{2}$ is given as

$$
\begin{aligned}
& a_{1}=-\left\langle\mathbf{c}^{2}\right\rangle+1=0 \\
& a_{2}=\frac{\left\langle\mathbf{c}^{4}\right\rangle}{2}-2\left\langle\mathbf{c}^{2}\right\rangle+1=\frac{\left\langle\mathbf{c}^{4}\right\rangle}{2}-1
\end{aligned}
$$

respectively. Here we have used the fact that substitution of $p=2$ into eq.(B3) leads to $\left\langle\mathbf{c}^{2}\right\rangle=1$. Substitution of $p=4$ into eq.(B3) leading to

$$
M_{4}=2 M_{2}\left\langle\mathbf{c}^{4}\right\rangle
$$

we obtain 


$$
4 M_{2}\left(a_{2}+1\right)-M_{4}=0 .
$$

On the other hand, the moments of the dimensionless collision integral $M_{p}$ is calculated as

$$
M_{p}=\int \mathrm{d} \mathbf{c} \int_{\mathbf{c} \cdot \hat{\sigma}>0} \mathrm{~d} \hat{\sigma}(\mathbf{c} \cdot \hat{\sigma}) F(\mathbf{c})\left(c^{p}-c^{\prime p}\right),
$$

where $c^{\prime}$ indicates the postcollision velocity of the tracer. From eq.(B13), we need the expression of $M_{2}$ and $M_{4}$ to obtain $a_{2}$. In general, it is impossible to obtain such the moments without the complete information of $F(\mathbf{c})$. Thus, we adopt an approximate method to obtain a closed relation for $M_{2}, M_{4}$ and $a_{2}$, i.e. we approximate the scaled velocity distribution function as $F(\mathbf{c})=\phi(\mathbf{c})\left\{1+a_{2} S_{2}\left(\mathbf{c}^{2}\right)\right\}$ using the expression of eq.(B) with $a_{p}=0$ for $p \geq 3$. Adopting such the approximation, the second moment is calculated as

$$
M_{2}=\sqrt{\pi}\left(1-e^{2}\right)\left(1+\frac{3}{8} a_{2}\right)
$$

The forth moment is calculated similarly as

$$
M_{4}=\sqrt{\pi}\left(1-e^{2}\right)\left(3+2 e^{2}\right)\left(1+\frac{15}{8} a_{2}\right)
$$

Inserting these moments into eq.(B13), we obtain

$$
a_{2}=\frac{1}{24}\left(1+30 e^{2} \pm \sqrt{-383+828 e^{2}+900 e^{4}}\right) \quad \text { if } \quad e \neq 1 .
$$

We adopt the stable solution of $a_{2}$ [9], i.e.

$$
a_{2}=\frac{1}{24}\left(1+30 e^{2}-\sqrt{-383+828 e^{2}+900 e^{4}}\right)
$$

for the inelastic case. Another solution of $a_{2}$ in eq.(B17) is unstable.

Here we discuss the stability of the coefficient $a_{2}$ in eq.(B17). To analyze the stability of $a_{2}$ we write eq.(10) in a more general form

$$
f(\mathbf{v}, t)=\frac{n}{v_{0}^{2}(t)} F(\mathbf{c}, t)
$$

which leads to 


$$
\frac{1}{d \rho_{0} v_{0}(t)} \frac{\partial\left\langle\mathbf{c}^{p}\right\rangle}{\partial t}-\frac{M_{2}}{2} p\left\langle\mathbf{c}^{p}\right\rangle+M_{p}=0
$$

in essentially the same way as eq.(B2). Using $F(\mathbf{c}, t)=\phi(\mathbf{c})\left\{1+a_{2}(t) S_{2}\left(\mathbf{c}^{2}\right)\right\}$ and $\left\langle\mathbf{c}^{2}\right\rangle=1$ which is bound, substitution of $p=4$ into eq. (B20) leads to

$$
\frac{\partial a_{2}(t)}{\partial t}=\frac{d \rho_{0} v_{0}(t)}{2}\left\{4 M_{2}\left(a_{2}(t)+1\right)-M_{4}\right\}
$$

for the coefficient $a_{2}(t)$. To discuss the linear stability of the coefficient $a_{2}(t)$, we divide it into the equilibrium part $\bar{a}_{2}$ and the time-dependent part $\delta a_{2}(t)$, i.e.

$$
a_{2}(t)=\bar{a}_{2}+\delta a_{2}(t)
$$

Note that $\bar{a}_{2}$ is $a_{2}$ in eq.(B18). Substituting eq.(B22) into eq.(B21) and ignoring the higher order $O\left(\delta a_{2}^{2}(t)\right)$ terms, we obtain

$$
\frac{\partial \delta a_{2}(t)}{\partial t}=\frac{d \rho_{0}}{2} \sqrt{\pi}\left(1-e^{2}\right) H(e) v_{0}(t) \delta a_{2}(t)
$$

which can be solved for $a_{2}(t)$ as

$$
\delta a_{2}(t)=\delta a_{2}(0) \exp \left[\frac{d \rho_{0}}{2} \sqrt{\pi}\left(1-e^{2}\right) H(e) \int_{0}^{t} v_{0}(t) \mathrm{d} t\right]
$$

where

$$
H(e) \equiv 3 \bar{a}_{2}-\left(\frac{15}{4} e^{2}+\frac{1}{8}\right)
$$

Thus, the stability of the coefficient $a_{2}(t)$ depends on the signs of $H(e)$, i.e. $a_{2}(t)$ is stable if $H(e)$ is negative. Figure 8 shows $H(e)$ as a function of the restitution coefficient $e$. Here we substitute the expressions of $a_{2}$ in eq. (B17) as $\bar{a}_{2}$. From Fig. 8, it is shown that $a_{2}$ in eq.(B18) is stable, while another $a_{2}$ in eq.(B17) is unstable.

It should be also mentioned that the elastic case $e=1$ is a singular point in eq.(B18), i.e. $F(\mathbf{c})=\phi(\mathbf{c})\left\{1+a_{2} S_{2}\left(\mathbf{c}^{2}\right)\right\}$ does not become the Gaussian even if $e=1$, and that eq.(B18) has real values only in the region

$$
\sqrt{-\frac{23}{50}+\frac{16 \sqrt{14}}{75}} \leq e \quad(<1) .
$$


The reason why $a_{2}$ does not exist in the inelastic Lorentz gas system of high inelasticity is thought to be that the solution by the Sonine expansion does not have terms of odd power of $\mathbf{c}$ and it can not express the scaled velocity distribution of the inelastic Lorentz gas system of high inelasticity by eq.(B4) even if we obtain the perfect form of eq.(B4) because its high energy tail is definitely exponential. It has been also demonstrated that the Sonine expansion diverges if the inelasticity is high in the inelastic Boltzmann gas system. [4] This means that we also can not express the scaled velocity distribution of the inelastic Boltzmann gas system of high inelasticity.

From eqs.(B15) and (B18), the expression of $M_{2}$ as a function of the restitution coefficient $e$ is given as

$$
M_{2}=\sqrt{\pi}\left(1-e^{2}\right)\left(\frac{65}{64}+\frac{15}{32} e^{2}-\frac{1}{64} \sqrt{-383+828 e^{2}+900 e^{4}}\right) .
$$

Judging from the expression of eq. (B15), the reason why $M_{2}$ is proportional not to $1-e^{2}$ but to $1-e$ is due to the deviation of the scaled velocity distribution function from the Gaussian, i.e. if $a_{2}=0$ in $F(\mathbf{c})=\phi(\mathbf{c})\left\{1+a_{2} S_{2}(\mathbf{c})\right\}, M_{2}$ in eq.(B15) becomes proportional to $1-e^{2}$ and the cooling rate of the inelastic Lorentz gas system becomes proportional to $1-e^{2}$ analytically. Note that because we multiply $a_{2}$ by $1-e^{2}$ in eq.(B15), $M_{2}$ holds even at the elastic case, i.e. $e=1$, though eq.(B18) does not hold at the elastic case.

\section{APPENDIX C: DIFFUSION COEFFICIENT $D_{E}(T)$}

We derive the expression of the diffusion coefficient $D_{e}(t)$ in eq.(15) explicitly by the method of the Sonine expansion.

Calculating eq.(9) in a similar way as eq.(B14), we obtain

$$
\int \mathrm{d} \mathbf{v} Y(\mathbf{v}) \Lambda X(\mathbf{v})=d \rho_{0} \int \mathrm{d} \mathbf{v} \int_{\mathbf{v} \cdot \hat{\sigma}>0} \mathrm{~d} \hat{\sigma}(\mathbf{v} \cdot \hat{\sigma}) X(\mathbf{v})\left[Y\left(\mathbf{v}^{\prime}\right)-Y(\mathbf{v})\right]
$$

where $\mathbf{v}^{\prime}$ is the postcollision velocity of the tracer:

$$
\mathbf{v}^{\prime}=\mathbf{v}-(1+e)(\mathbf{v} \cdot \hat{\sigma}) \hat{\sigma}
$$


Here we approximate $\mathbf{B}(\mathbf{v}, T(t))$ in the first Sonine approximation as

$$
\mathbf{B}(\mathbf{v}, T(t)) \sim \mathbf{v} f_{a_{2}}(\mathbf{v}, t)
$$

where the velocity distribution function of the tracer $f(\mathbf{v}, t)$ is approximated as

$$
\begin{aligned}
f(\mathbf{v}, t) & \simeq f_{a_{2}}(\mathbf{v}, t) \\
& \equiv \frac{m}{2 \pi T(t)} \exp \left[-\frac{m}{2 T(t)} \mathbf{v}^{2}\right]\left\{1+a_{2}\left(1-\frac{m}{T(t)} \mathbf{v}^{2}+\frac{m^{2}}{8 T^{2}(t)} \mathbf{v}^{4}\right)\right\}
\end{aligned}
$$

Use of eq.(C1) into eq.(43) leads to

$$
\nu_{D}=\frac{d \rho_{0}(1+e)}{2} \sqrt{\frac{2 \pi T(t)}{m}}\left(2+\frac{3}{4} a_{2}\right)
$$

Substitution of this expression of $\nu_{D}$ into eq. (45) leads to

$$
D_{e}(t)=\sqrt{\frac{2 T(t)}{\pi m}} \frac{1}{d \rho_{0}} \frac{1}{(e+1)^{2}\left(1+\frac{3}{8} a_{2}\right)} .
$$

This is the the expression of the diffusion coefficient $D_{e}(t)$ in eq. (45) by the method of the Sonine expansion.

However, the diffusion coefficient $D_{e}(t)$ by the method of the Sonine expansion does not work in the Lorentz gas system as follows. From the definition of the diffusion coefficient:

$$
\left\langle\mathbf{r}^{2}(t)\right\rangle_{e}=4 \int_{0}^{t} D_{e}(t) \mathrm{d} t^{\prime}
$$

substitution of eq.(C7) into this leads to the mean square displacement of the tracer by the method of the Sonine expansion as

$$
\left\langle\mathbf{r}^{2}(t)\right\rangle_{e}=\frac{8}{\pi d^{2} \rho_{0}^{2}} \frac{1}{(1+e)^{2}\left(1-e^{2}\right)\left(1+\frac{3}{8} a_{2}\right)^{2}} \ln \left[1+\sqrt{\frac{\pi}{2}} d \rho_{0}\left(1-e^{2}\right)\left(1+\frac{3}{8} a_{2}\right) t\right] .
$$

Thus, we have derived the logarithmic time dependence of the mean square displacement in terms of a standard method of gas kinetics.

Figure 9 shows $\left\langle\mathbf{r}^{2}(t)\right\rangle_{e} \times(1+e)^{2}\left(1-e^{2}\right)\left(1+\frac{3}{8} a_{2}\right)^{2}$ versus $\left(1-e^{2}\right)\left(1+\frac{3}{8} a_{2}\right) t$ plots with $a_{2}$ in eq.(B18) for $e=0.6,0.7$ and 0.9. Because $a_{2}$ for $e=0.4$ does not exist, we can not 
plot the results of our simulation for $e=0.4$. It should be mentioned that the results of our simulation shown as the symbols are not on a same curve. Equation (C9) shown as the broken line deviates from the results of our simulation. Thus, we suggest that the diffusion coefficient $D_{e}(t)$ by the method of the Sonine expansion is not a good approximation in the Lorentz gas system.

\section{APPENDIX D: THE DERIVATION OF DIFFUSION COEFFICIENT $D_{E}(V, T)$}

From the fact the velocity of the tracer $\mathbf{v}$ is distributed, the diffusion coefficient of the tracer is expected to depend on the velocity of the tracer $\mathbf{v}$. We derive the expression of the diffusion coefficient $D_{e}(V, t)$ in eq.(48) which depends on the magnitude of the scaled hopping velocity $V$ as follows.

We derive the diffusion equation which depends on the velocity of the tracer by almost similar means which we have adopted to obtain eq.(46), i.e. by the means of a ChapmanEnskog expansion in the density gradient of the tracer. [10,11]

Integrating eq. (24) by $\mathbf{v}$, we obtain

$$
\int 2 \pi v\left[\frac{\partial n(\mathbf{r}, v, t)}{\partial t}+\nabla \cdot \mathbf{J}(\mathbf{r}, v, t)\right] \mathrm{d} v=0
$$

where the density of the tracer $n(\mathbf{r}, v, t)$ is defined as

$$
n(\mathbf{r}, v, t) \equiv \int p(\mathbf{r}, v \hat{\mathbf{v}}, t) \delta(|\hat{\mathbf{v}}|-1) \mathrm{d} \hat{\mathbf{v}}
$$

and the flux of the tracer $\mathbf{J}(\mathbf{r}, v, t)$ is defined as

$$
\mathbf{J}(\mathbf{r}, v, t) \equiv \int v \hat{\mathbf{v}} p(\mathbf{r}, v \hat{\mathbf{v}}, t) \delta(|\hat{\mathbf{v}}|-1) \mathrm{d} \hat{\mathbf{v}}
$$

Note that $\mathbf{v}=v \hat{\mathbf{v}}$ with $v=|\mathbf{v}|$. Because eq.(D1) holds for each speed of the tracer $v$, we obtain the equation of continuity which depends on $v$ as

$$
\frac{\partial n(\mathbf{r}, v, t)}{\partial t}+\nabla \cdot \mathbf{J}(\mathbf{r}, v, t)=0
$$


Here we adopt the Chapman-Enskog method in which the position-velocity distribution of the tracer $p(\mathbf{r}, \mathbf{v}, t)$ is written as a series expansion in a formal parameter $\epsilon$ as in eq.(29). Equation (30) is now replaced by

$$
\frac{\partial n(\mathbf{r}, v, t)}{\partial t}=\sum_{j=0}^{\infty} \epsilon^{j+1} \partial_{t}^{(j)} n(\mathbf{r}, v, t)
$$

with

$$
\partial_{t}^{(j)} n(\mathbf{r}, v, t)=-\nabla^{(0)} \cdot \mathbf{J}^{(j)}(\mathbf{r}, v, t)
$$

and

$$
\mathbf{J}^{(j)}(\mathbf{r}, v, t)=\int v \hat{\mathbf{v}} p^{(j)}(\mathbf{r}, v \hat{\mathbf{v}}, t) \delta(|\hat{\mathbf{v}}|-1) \mathrm{d} \hat{\mathbf{v}} .
$$

Taking into account eq.(33), the zeroth order of eq.(24) becomes eq.(34). Here eq.(35) is replaced by

$$
p^{(0)}(\mathbf{r}, \mathbf{v}, t)=\frac{n(\mathbf{r}, v, t)}{m(v, t)} f(\mathbf{v}, t),
$$

where

$$
m(v, t) \equiv \int f(v \hat{\mathbf{v}}, t) \delta(|\hat{\mathbf{v}}|-1) \mathrm{d} \hat{\mathbf{v}}
$$

From eqs. (D7) and (D8),

$$
\mathbf{J}^{(0)}(\mathbf{r}, v, t)=v \frac{n(\mathbf{r}, v, t)}{m(v, t)} \int \hat{\mathbf{v}} f(v \hat{\mathbf{v}}, t) \delta(|\hat{\mathbf{v}}|-1) \mathrm{d} \hat{\mathbf{v}}=0,
$$

i.e. the flux of the tracer vanishes to zeroth order, which leads to $\partial_{t}^{(0)} n(\mathbf{r}, v, t)=0$. Additionally, from eq.(D8), $\partial p^{(0)}(\mathbf{r}, \mathbf{v}, t) / \partial n(\mathbf{r}, v, t)=f(\mathbf{v}, t) / m(v, t)$.

To the first order in the density gradient $\epsilon$, eq.(36) is replaced by

$$
\begin{aligned}
& \left(\partial_{t}^{(0)} n(\mathbf{r}, v, t)\right) \frac{\partial p^{(0)}(\mathbf{r}, \mathbf{v}, t)}{\partial n(\mathbf{r}, v, t)}+\frac{\partial T(t)}{\partial t} \frac{\partial p^{(1)}(\mathbf{r}, \mathbf{v}, t)}{\partial T(t)} \\
& +\left(\mathbf{v} \cdot \nabla^{(0)} n(\mathbf{r}, v, t)\right) \frac{\partial p^{(0)}(\mathbf{r}, \mathbf{v}, t)}{\partial n(\mathbf{r}, v, t)}=\Lambda p^{(1)}(\mathbf{r}, \mathbf{v}, t) .
\end{aligned}
$$

From the results of the zeroth order and eq.(5) with $l=1 / 2 d \rho_{0}$, i.e. 


$$
\frac{d T(t)}{d t}=-\frac{v_{0}(t)}{l} h(e) T(t)
$$

eq.(D11) becomes

$$
\left(\frac{v_{0}(t)}{l} h(e) T(t) \frac{\partial}{\partial T(t)}+\Lambda\right) p^{(1)}(\mathbf{r}, \mathbf{v}, t)=\left(\mathbf{v} \cdot \nabla^{(0)} n(\mathbf{r}, v, t)\right) \frac{f(\mathbf{v}, t)}{m(v, t)} .
$$

Similar to eq.(38), the solution $p^{(1)}(\mathbf{r}, \mathbf{v}, t)$ is proportional to $\nabla^{(0)} n(\mathbf{r}, v, t)$ :

$$
p^{(1)}(\mathbf{r}, \mathbf{v}, t)=\mathbf{B}(\mathbf{v}, T(t)) \cdot \nabla^{(0)} n(\mathbf{r}, v, t),
$$

where the vector $\mathbf{B}(\mathbf{v}, T(t))$ also depends on $t$ through $T(t)$. Substitution of this into eq.(D13) leads to

$$
\left(\frac{v_{0}(t)}{l} h(e) T(t) \frac{\partial}{\partial T(t)}+\Lambda\right) \mathbf{B}(\mathbf{v}, T(t))=\mathbf{v} \frac{f(\mathbf{v}, t)}{m(v, t)} .
$$

Here the contribution to the particle flux of first order in the density gradient is given by

$$
\mathbf{J}^{(1)}(\mathbf{r}, v, t)=-D_{e}(v, t) \nabla^{(0)} n(\mathbf{r}, v, t) .
$$

with

$$
D_{e}(v, t) \equiv-\frac{v}{2} \int \hat{\mathbf{v}} \cdot \mathbf{B}(v \hat{\mathbf{v}}, T(t)) \delta(|\hat{\mathbf{v}}|-1) \mathrm{d} \hat{\mathbf{v}}
$$

Note that we have used symmetry considerations in two-dimension. $D_{e}(v, t)$ is the diffusion coefficient which depends on the speed of the tracer $v$.

Using this diffusion coefficient $D_{e}(v, t)$ and eq.(D9), eq.(D15) becomes

$$
\left(\frac{v_{0}(t)}{l} h(e) T(t) \frac{\partial}{\partial T(t)}-\nu_{D}\right) D_{e}(v, t)=-\frac{v^{2}}{2} .
$$

with

$$
\nu_{D} \equiv-\frac{\int(\Lambda \mathbf{B}(v \hat{\mathbf{v}}, T(t)) \cdot v \hat{\mathbf{v}} \delta(|\hat{\mathbf{v}}|-1) \mathrm{d} \hat{\mathbf{v}}}{\int v \hat{\mathbf{v}} \cdot \mathbf{B}(v \hat{\mathbf{v}}, T(t)) \delta(|\hat{\mathbf{v}}|-1) \mathrm{d} \hat{\mathbf{v}}}
$$

Dimensional analysis requires $D_{e}(v, t) \sim v_{0}^{-1}(t) \sim T^{-\frac{1}{2}}(t)$. Therefore, 


$$
\frac{\partial D_{e}(v, t)}{\partial T(t)}=-\frac{D_{e}(v, t)}{2 T(t)}
$$

Substitution of this into eq.(D18) becomes

$$
D_{e}(v, t)=\frac{v^{2}}{2}\left(\frac{h(e) v_{0}(t)}{2 l}+\nu_{D}\right)^{-1} \sim \frac{v^{2} l}{v_{0}(t)},
$$

where we assume $\nu_{D} \sim v_{0}(t) / l$ from eq.(D19). Assuming the hopping velocity $\mathbf{l} / \tau$ corresponds to the velocity of the tracer $\mathbf{v}$, we obtain the diffusion coefficient eq.(48) which depends on the magnitude of the scaled hopping velocity $V$ as

$$
D_{e}(V, t)=\kappa \frac{V^{2} l}{\alpha+\beta t}
$$

where $\kappa$ is an unimportant dimensionless constant. 


\section{REFERENCES}

[1] C.S. Campbell: Ann. Rev. Fluid Mech. 22 (1990) 57.

[2] see e.g. L. P. Kadanoff: Rev. Mod. Phys. 71 (1999) 435.

[3] J. J. Brey, D. Cubero and M. J. Ruiz-Montero: Phys. Rev. E 59 (1999) 1256.

[4] M. Huthmann, J. A. G. Orza and R. Brito: Granular Matter 2 (2000) 189.

[5] T. P. C. van Noije and M.H. Ernst: Granular Matter 1 (1998) 57.

[6] S. E. Esipov and T. Pöschel: J. Stat. Phys. 86 (1997) 1385.

[7] J. J. Brey, M.J. Ruiz-Montero and D.Cubero: Phys. Rev. E 54 (1996) 3664.

[8] J. M. Montanero, A. Santos: Granular Matter 2 (2000) 53.

[9] N. V. Brilliantov and T. Pöschel: Phys. Rev. E 61 (2000) 2809.

[10] see e.g. S. Chapmann and T. G. Cowling: The mathematical theory of non-uniform gases(Cambridge University Press, London, 1990).

[11] J. J. Brey, M. J. Ruiz-Montero, D. Cubero and R. Garcia-Rojo: Phys. Fluids 12 (2000) 876.

[12] S. Chen, Y. Deng, X. Nie and Y. Tu: Phys. Lett. A 269 (2000) 218.

[13] P. Deltour and J.-L. Barrat: J. Phys. I (Paris) 7 (1997) 137.

[14] I. Goldhrirsch and G. Zanetti: Phys. Rev. Lett. 70 (1993) 1619.

[15] S. McNamara and V. R. Young: Phys. Fluids. A 5 (1993) 34.

[16] N. F. Carnahan and K. E. Staring: J. Chem. Phys. 51 (1969) 635.

[17] D. R. Wilkinson and S. F. Edwards: Proc. R. Soc. (London), Ser A 381 (1982) 33.

[18] P. A. Martin and J. Piasecki: Physica A 265 (1999) 19. 
[19] see e.g. D. J. Jackson: Classical Electrodynamics, New York (1999).

[20] L. Hansen, M. Christensen and E. Mosekilde: Phys. Scr. 51 (1995) 35.

[21] see e.g. D. C. Rapaport: J. Comput. Phys. 34 (1980) 184.

[22] see e.g. M. P. Allen and D. J. Tildesley: Computer Simulation of Liquid,(Clarendon Press, Oxford, 1989).

[23] M. Isobe: Int. J. Mod. Phys. C 10 (1999) 1281.

[24] P. K. Haff: J. Fluid Mech. 134 (1983) 401. 


\section{FIGURES}

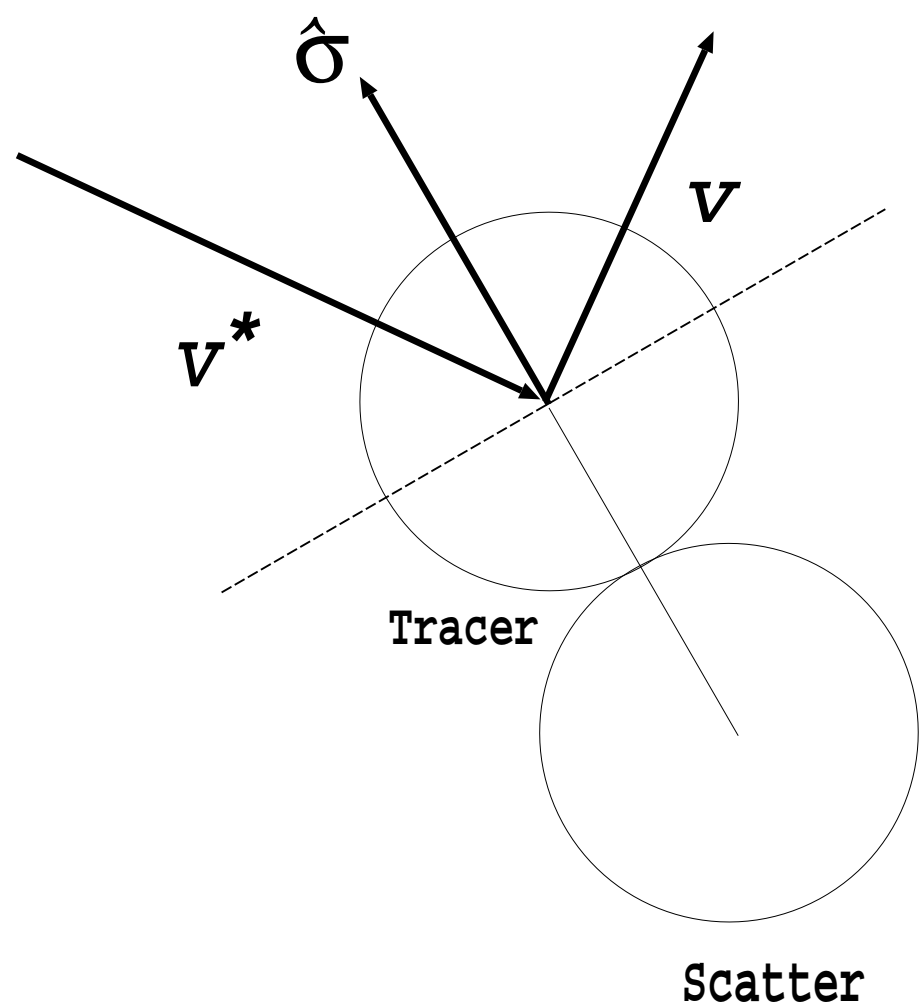

FIG. 1. Unit vector $\hat{\sigma}$ pointing from the center of a scatter to the center of a tracer. The precollision velocity of the tracer and the postcollision velocity of it are expressed as $\mathbf{v}^{*}$ and $\mathbf{v}$, respectively. 


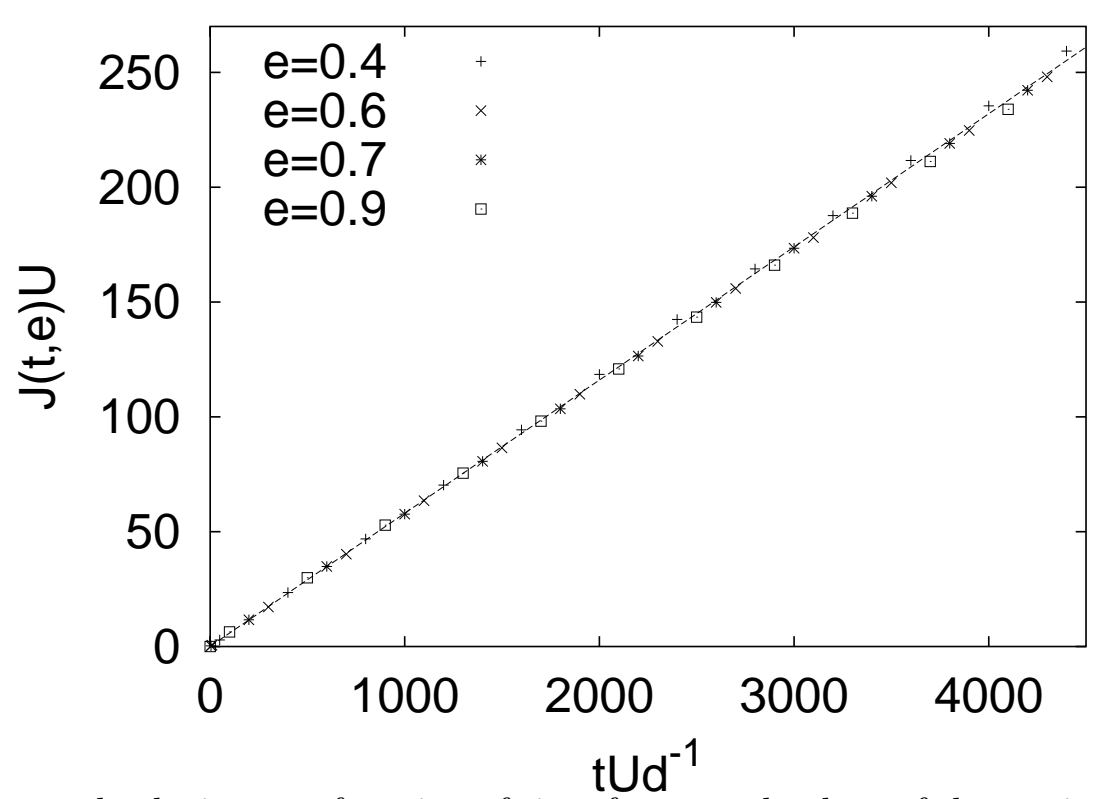

FIG. 2. Thermal velocity as a function of time for several values of the restitution coefficient $e$. Note that $J(t, e) \equiv\left(v_{0}^{-1}(t)-v_{0}^{-1}(0)\right) /(1-e)$. The straight line $0.058 \times t U d^{-1}$ shown as the broken line is the best fitting function of the results of our simulation. 


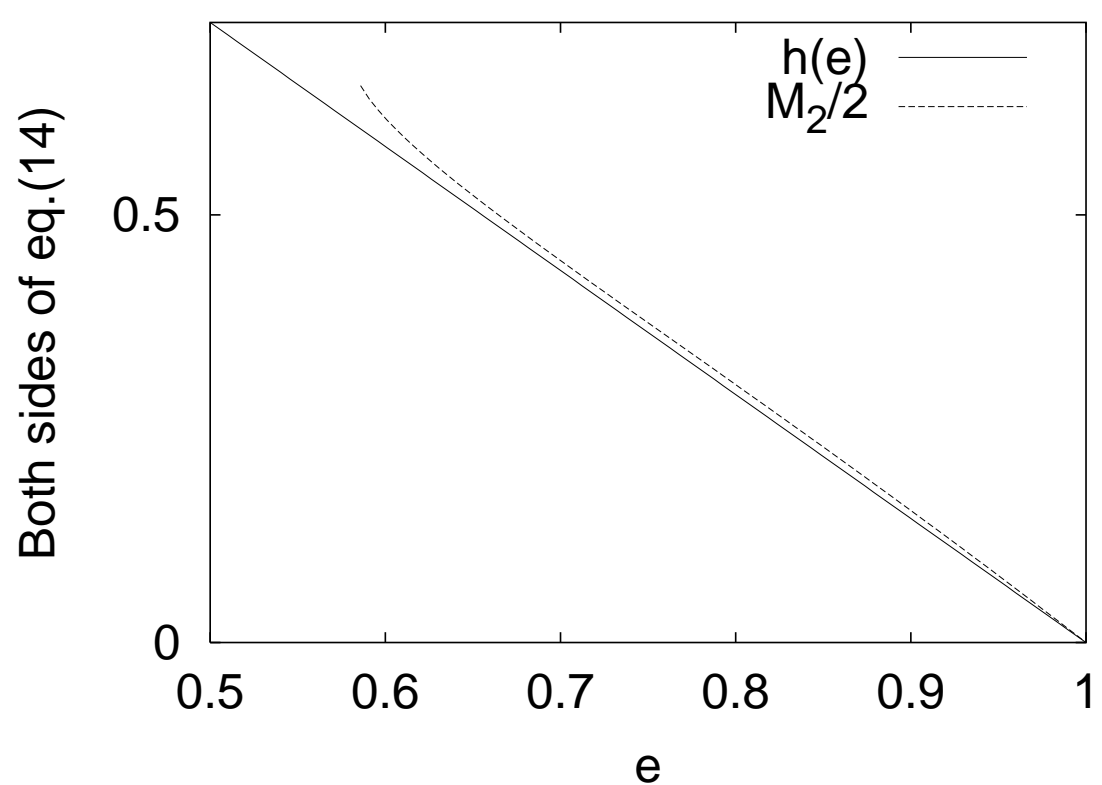

FIG. 3. $M_{2} / 2$ (short-dashed line) and $h(e)$ (solid line) as a function of the restitution coefficient e. $M_{2} / 2$ is obtained from eq.(B27), while $h(e)$ is obtained from eq.(8) which has been estimated from the data of our simulation. 


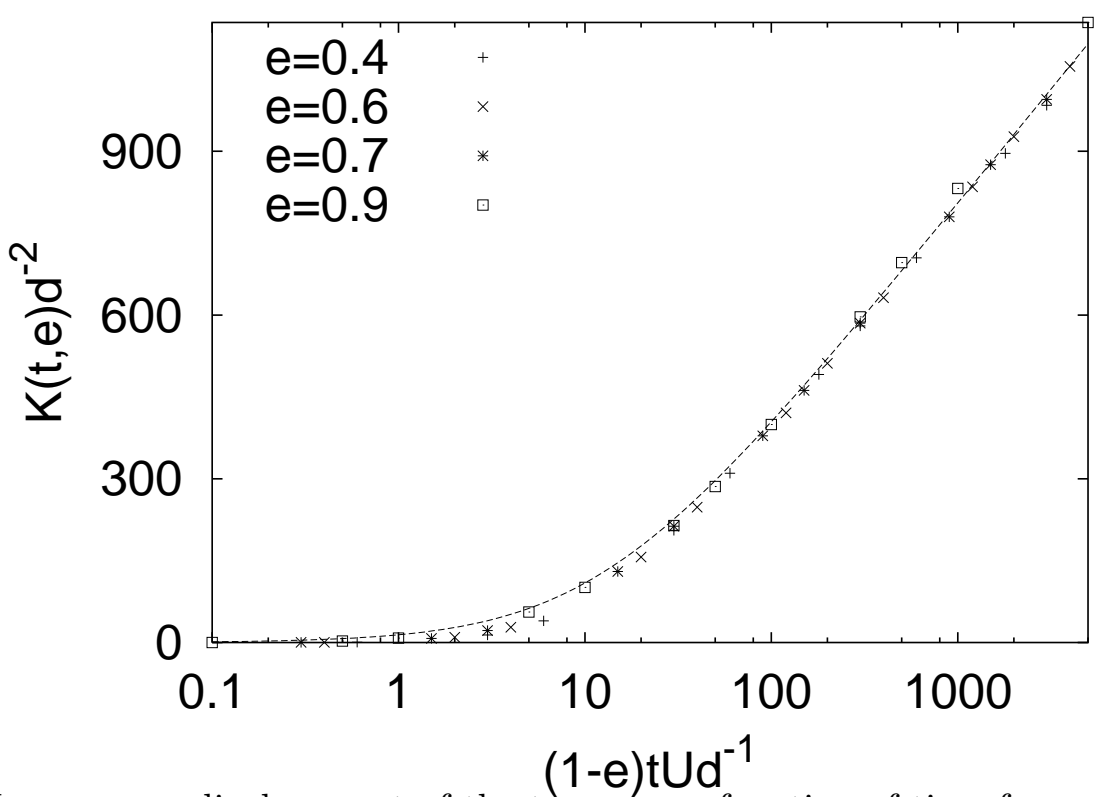

FIG. 4. Mean square displacement of the tracer as a function of time for several values of the restitution coefficient $e$. Note that $K(t, e) \equiv\left\langle\mathbf{r}^{2}(t)\right\rangle_{e} \times \ln (1+l \beta)$. The symbols are results from our simulation. The broken line is obtained from eq.(22) with $l \simeq 13.5 d$ and $\beta \simeq 0.058 \times(1-e) d^{-1}$. 


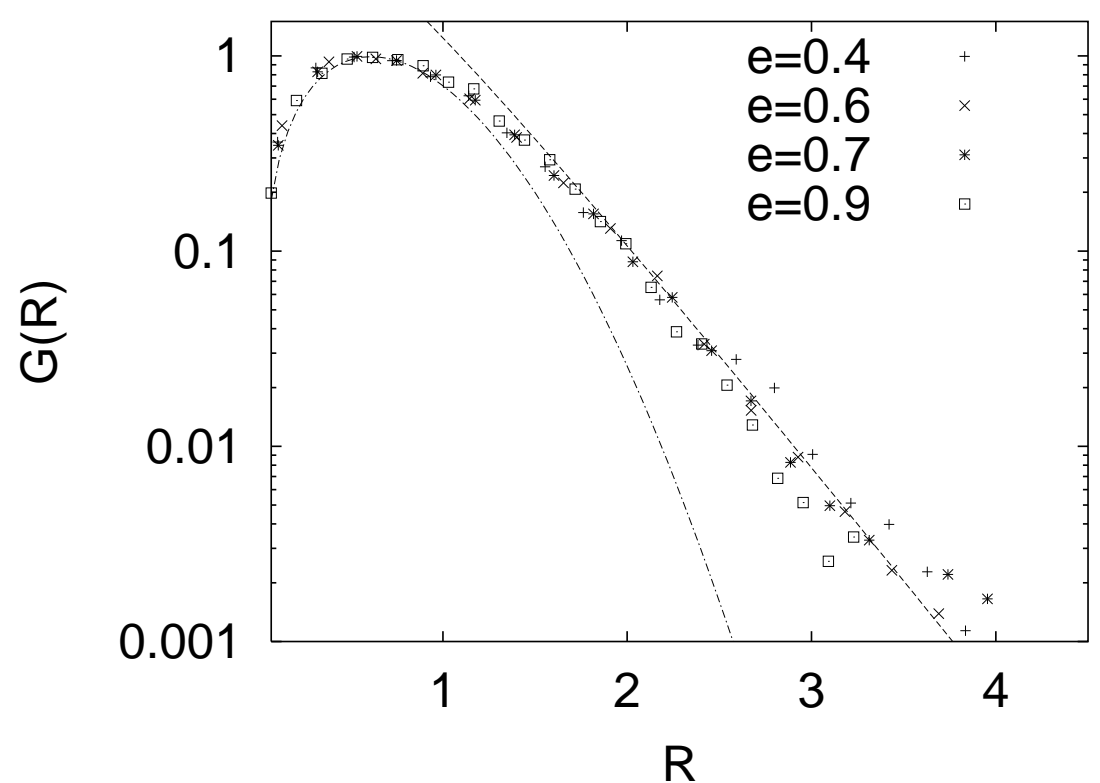

FIG. 5. Scaled position distribution of the tracer $G(R)=\sqrt{\left\langle\mathbf{r}^{2}(t)\right\rangle_{e}} \times g_{e}(r, t)$ as a function of the scaled distance $R \equiv r / \sqrt{\left\langle\mathbf{r}^{2}(t)\right\rangle_{e}}$ from the center of the system at which the tracer started. The symbols are the data of our simulation which are plotted for several values of the restitution coefficient $e=0.4,0.6,0.7$ and 0.9 at four different times $t=3000 d U^{-1}, 300 d U^{-1}, 500 d U^{-1}$ and $1000 d U^{-1}$, respectively. The broken line and the dash-dotted line are the approximate solutions of $G(R)$. The simulation data in the tail region correspond to eq. 58 with $\eta=0.25$ and $\gamma \simeq 7.39$, while the simulation data in the peak region correspond to eq.(59) with $\eta=0.75$ and $\bar{V}=1 / \sqrt{2}$. 


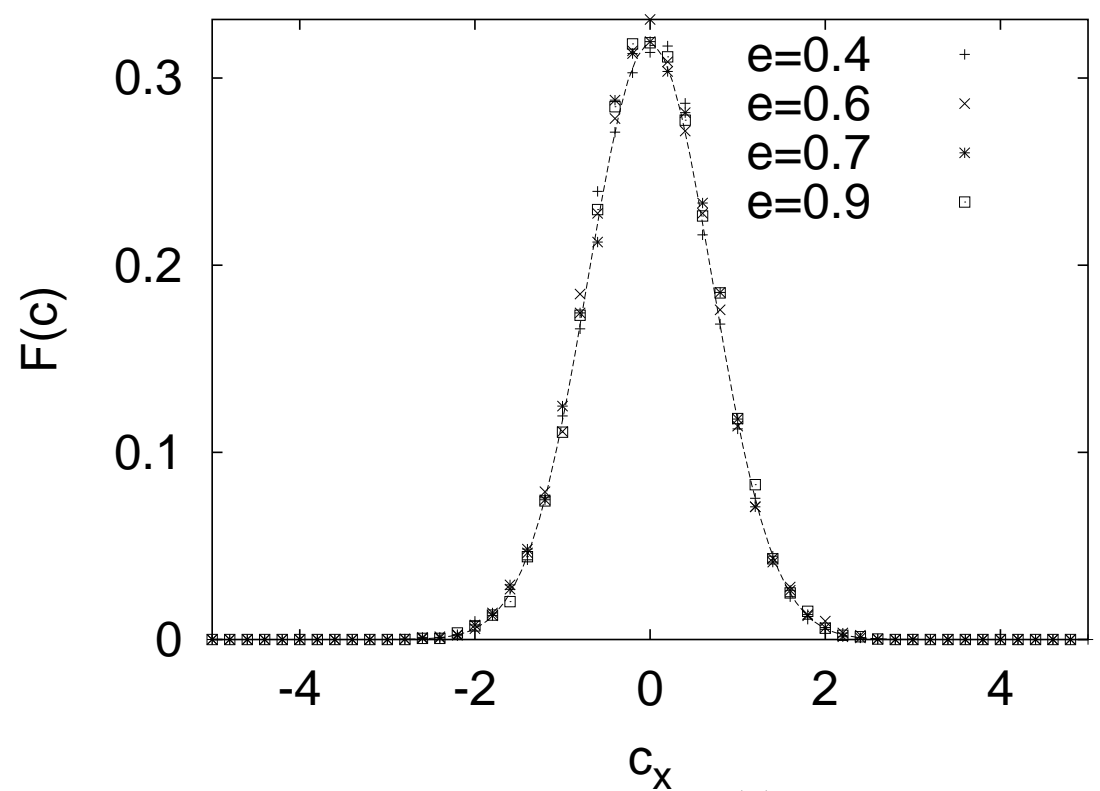

FIG. 6. Scaled velocity distribution of the tracer $F(\mathbf{c})$ as a function of $c_{x}$. The symbols are data of our simulation. The data are plotted for several values of the restitution coefficient $e=0.4,0.6,0.7$ and 0.9 at four different times $t=3000 d U^{-1}, 300 d U^{-1}, 500 d U^{-1}$ and $1000 d U^{-1}$, respectively. The broken line is the Maxwell-Boltzmann distribution $\pi^{-1} \mathrm{e}^{-\mathbf{c}^{2}}$. 


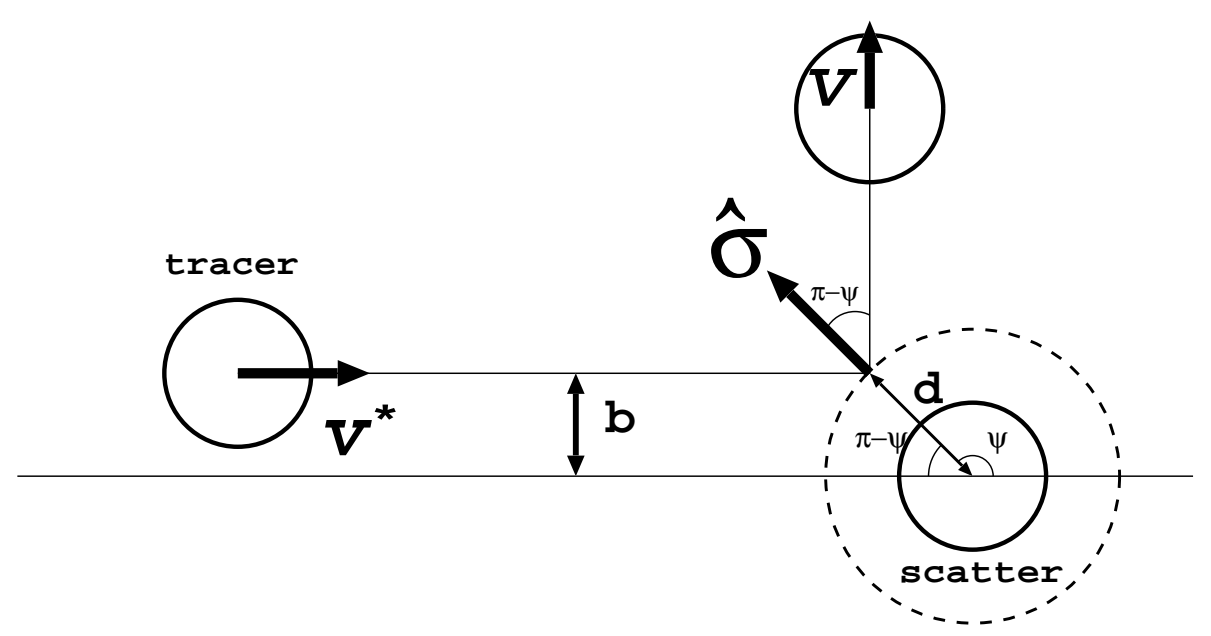

FIG. 7. Schematic description of a collision. 


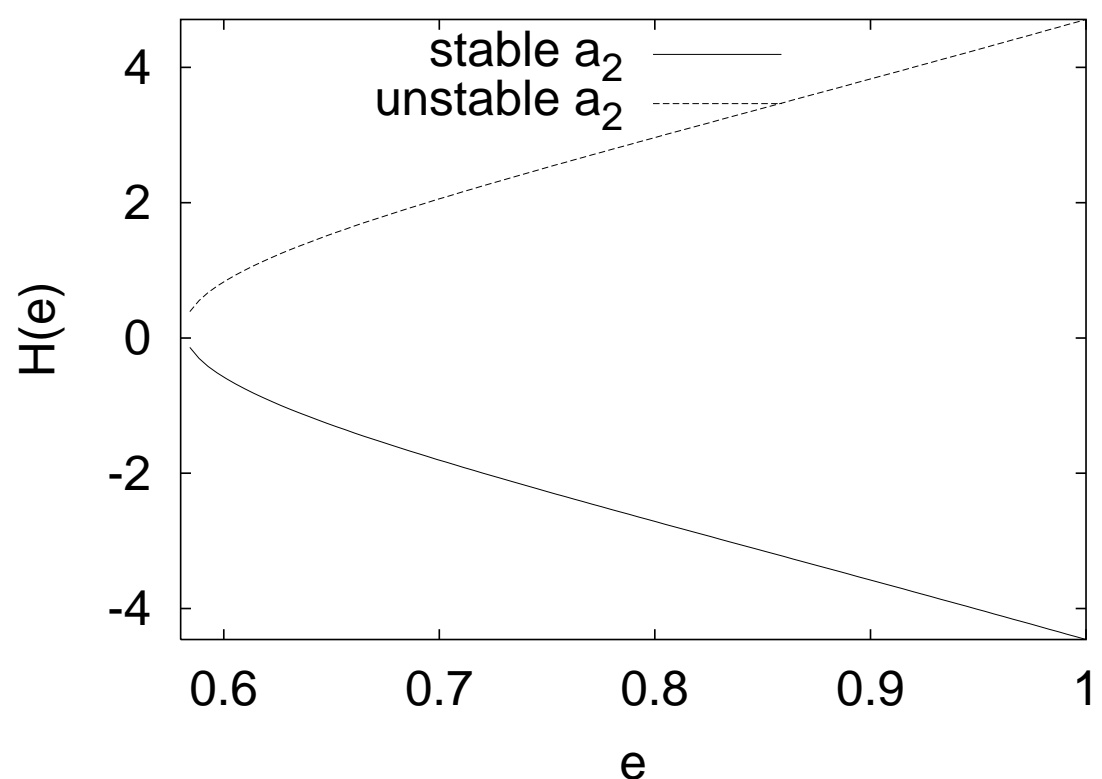

FIG. 8. $H(e)$ as a function of $e$. The solid line is $a_{2}$ in eq.(B18), while the broken line is another $a_{2}$ in eq.(B17). 


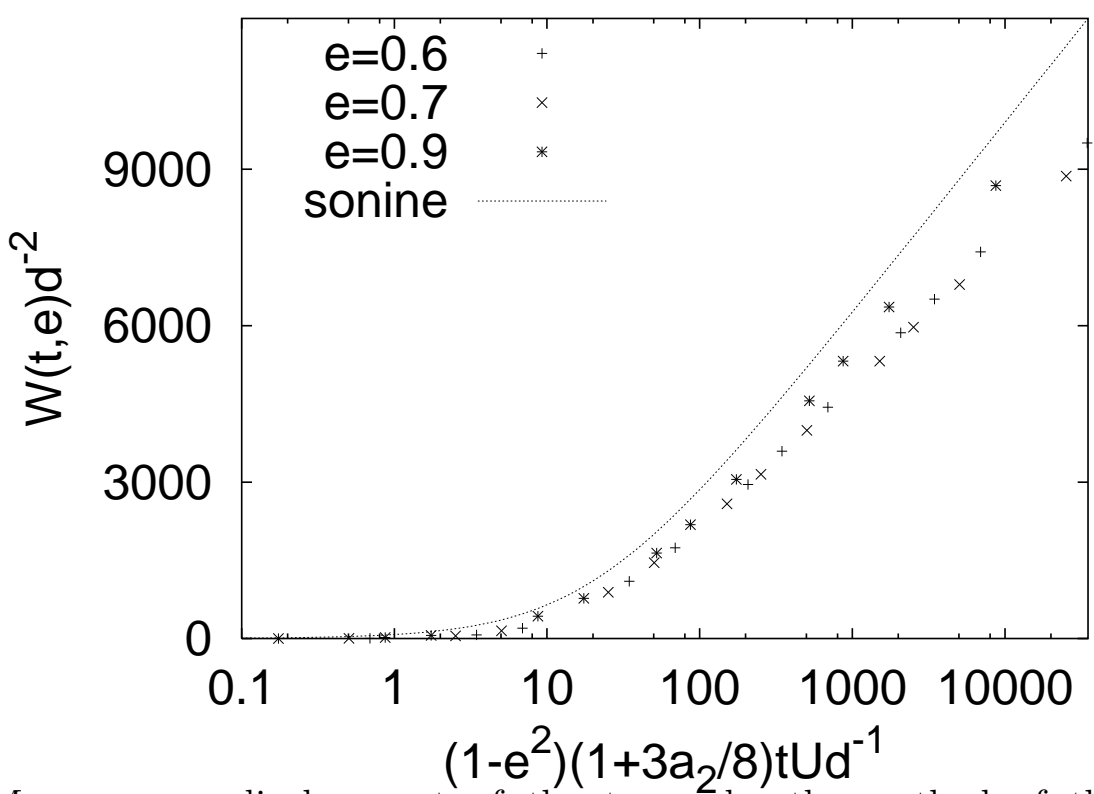

FIG. 9. Mean square displacement of the tracer by the method of the Sonine expansion as a function of time for several values of the restitution coefficient $e$. Note that $W(t, e) \equiv\left\langle\mathbf{r}^{2}(t)\right\rangle_{e} \times(1+e)^{2}\left(1-e^{2}\right)\left(1+\frac{3}{8} a_{2}\right)^{2}$ with $a_{2}$ in eq.(B18). The symbols are results from our simulation. The broken line is obtained from eq.(C9) with $a_{2}$ in eq.(B18). 